\title{
LA-UR-21-28556
}

Approved for public release; distribution is unlimited.

Title: $\quad$ End-to-End Modeling of a Kilonova

Author(s): $\quad$ Miller, Jonah Maxwell

Intended for: Departmental Colloquium

Issued: $\quad$ 2021-08-26 
Disclaimer:

Los Alamos National Laboratory, an affirmative action/equal opportunity employer, is operated by Triad National Security, LLC for the National Nuclear Security Administration of U.S. Department of Energy under contract 89233218CNA000001. By approving this article, the publisher recognizes that the U.S. Government retains nonexclusive, royalty-free license to publish or reproduce the published form of this contribution, or to allow others to do so, for U.S. Government purposes. Los Alamos National Laboratory requests that the publisher identify this article as work performed under the auspices of the U.S. Department of Energy. Los Alamos National Laboratory strongly supports academic freedom and a researcher's right to publish; as an institution, however, the Laboratory does not endorse the viewpoint of a publication or guarantee its technical correctness. 


\section{End-to-End Modeling of a Kilonova}

Jonah M. Miller, in collaboration with:

S. De, K. Lund, S. Curtis, T. Sprouse,

N. Wolfe, V. U-Hurtado,

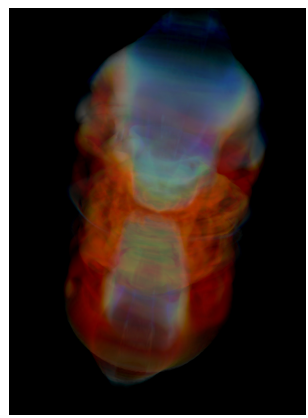

And Many More...

Los Alamos National Laboratory

UNAM Gravity Seminar 


\section{Cosmic Gold}

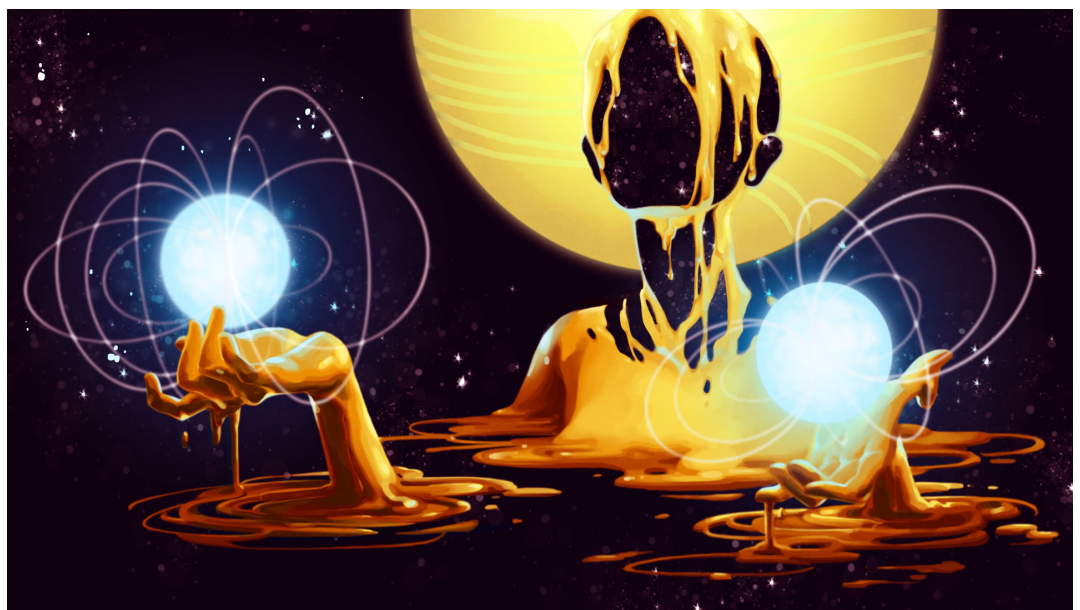

Ashley Mackenzie for Quanta Magazine, March 23, 2017 


\section{The 170817 Merger}

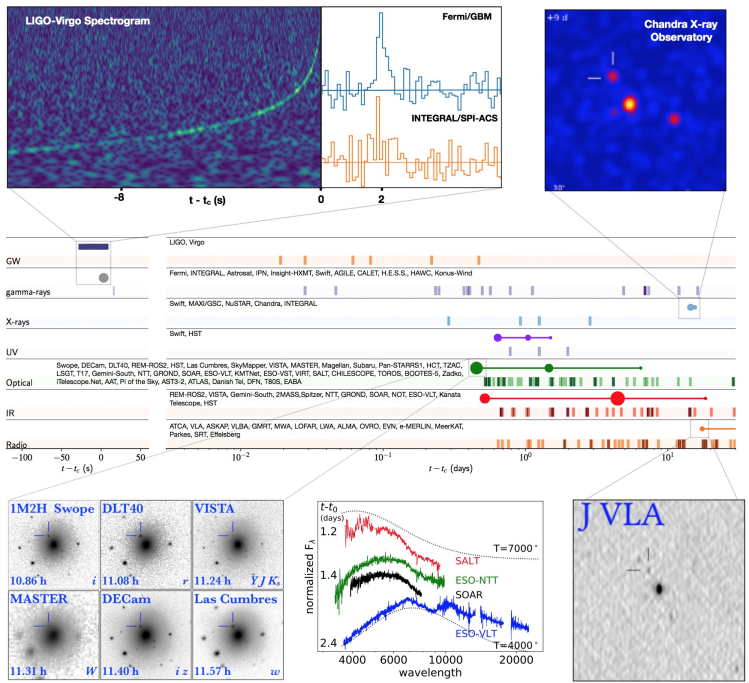

Abbot+, 2017 


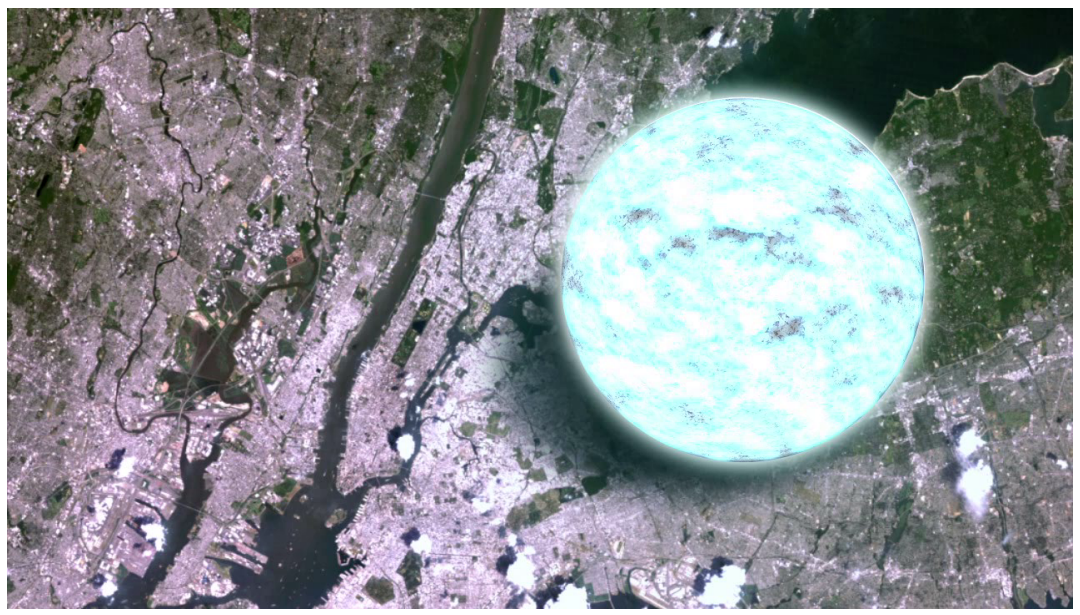

Wikimedia Commons 


\section{Outline of Talk}

- An overview of the process of forward modeling, from end to end - Emphasis on the disk, and neutrino transport

- A deeper look into a related system, a collapsar disk, and what it tells us about mergers 
Neutron Star Mergers: A 2+ Component Model
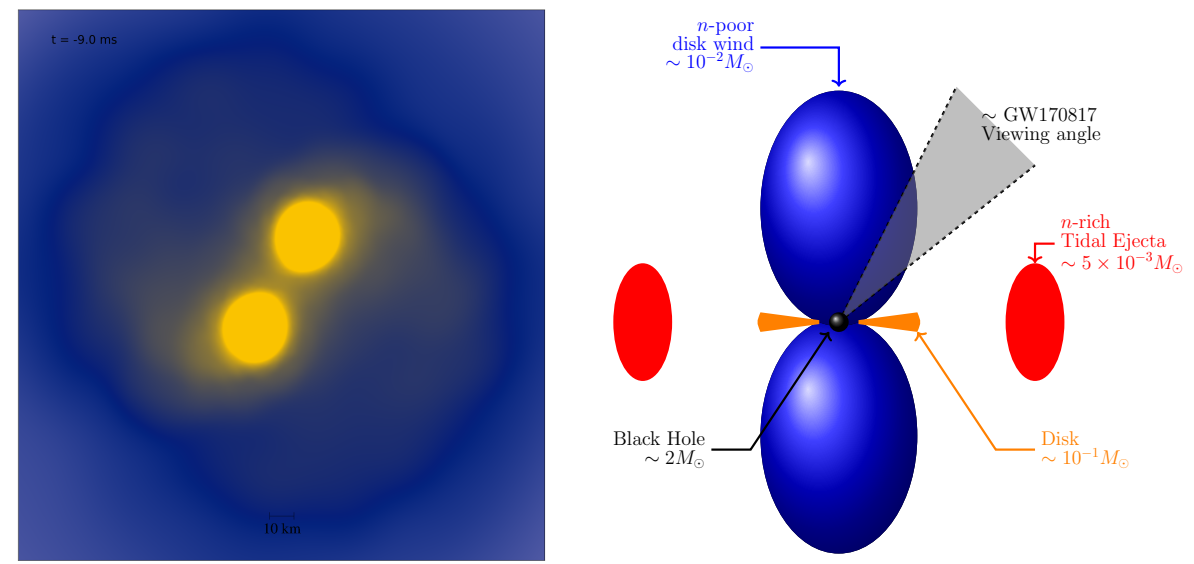

Co-design summer school, 2016 

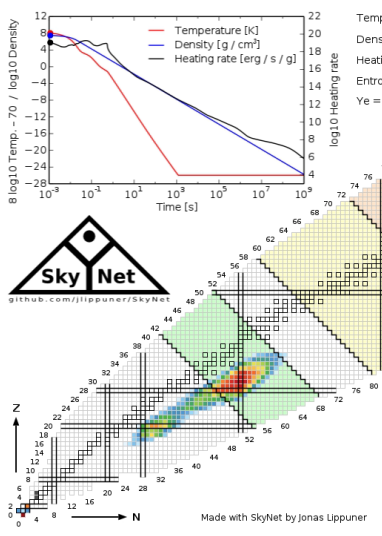

Made with SkyNet by Jonas Lippuner
Temperature $=5.68 \mathrm{E}+09 \mathrm{~K}$

Density $=2.98 \mathrm{E}+07 \mathrm{~g} / \mathrm{cm}^{3}$

Heating rate $=1.09 \mathrm{E}+19 \mathrm{erg} / \mathrm{s} / \mathrm{g}$

Entropy $=9.99 \mathrm{E}+00 \mathrm{~kg} /$ baryon

$Y e=0.130$

Y.130
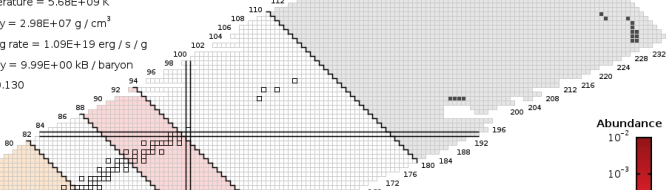

Abundance

$\square$ Unstable nuclide

- Stable nuclide

- Missing nuclide

" Closed neutron shell

= closed proton shell

Time $=1.13 \mathrm{E}-03 \mathrm{~s}$

$=1.1 \mathrm{~ms}$

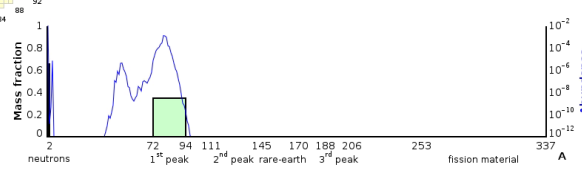

\section{Courtesy of J. Lippuner}



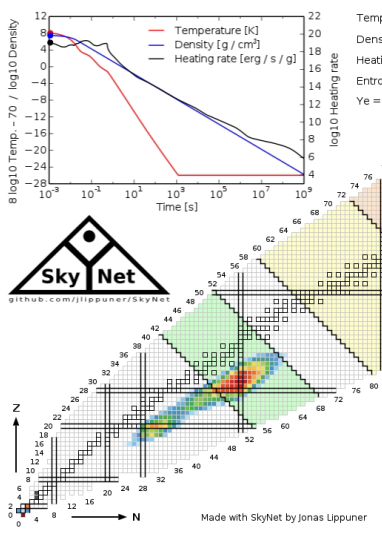

Made with SkyNet by Jonas Lippuner

Temperature $=5.68 \mathrm{E}+09 \mathrm{~K}$

Density $=2.98 \mathrm{E}+07 \mathrm{~g} / \mathrm{cm}^{3}$

Heating rate $=1.09 \mathrm{E}+19 \mathrm{erg} / \mathrm{s} / \mathrm{g}$

Entropy $=9.99 \mathrm{E}+00 \mathrm{~kg} /$ baryon

$Y e=0.130$

Y.130
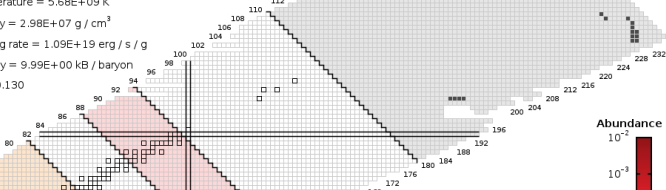

Abundance

$\square$ Unstable nuclide

- Stable nuclide

- Missing nuclide

" Closed neutron shell

= closed proton shell

Time $=1.13 \mathrm{E}-03 \mathrm{~s}$

$=1.1 \mathrm{~ms}$

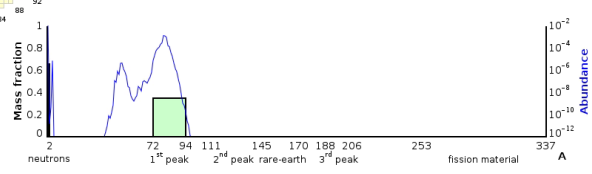

$10^{-10}$

Courtesy of J. Lippuner 


\section{Opacity}

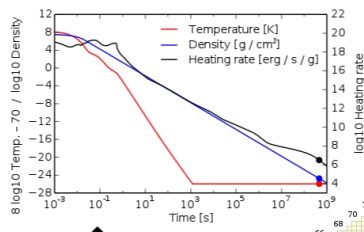

Temperature $=3.20 \mathrm{E}+05 \mathrm{~K}$ Density $=1.74 \mathrm{E}-25 \mathrm{~g} / \mathrm{cm}^{3}$

Heating rate $=3.16 \mathrm{E}+06 \mathrm{erg} / \mathrm{s} / \mathrm{g}$

Entropy $=1.28 \mathrm{E}+03 \mathrm{kB} /$ baryon

Ye $=0.403$

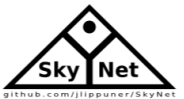

몽

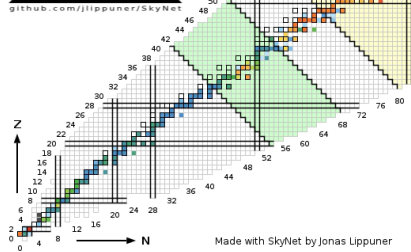

Made with SkyNet by Jonas Lippuner

$=0.403$
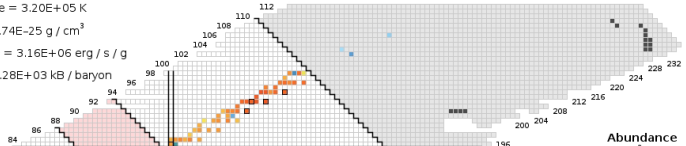

$m$ 212216

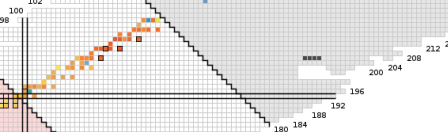

Abundance

Unstable nuclide

Stable nuclide

- Missing nuclide

1" Closed neutron shell

= closed proton shell

Time $=4,59 \mathrm{E}+08 \mathrm{~s}$

$=15 \mathrm{yr}$

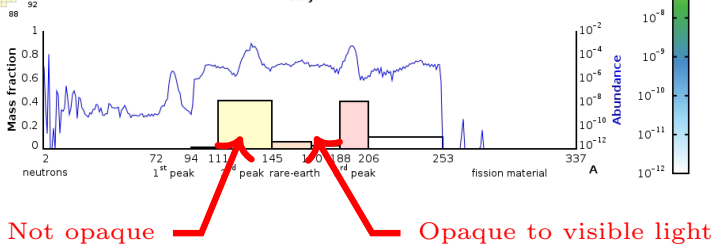


SSS17a

C. August 17, 2017

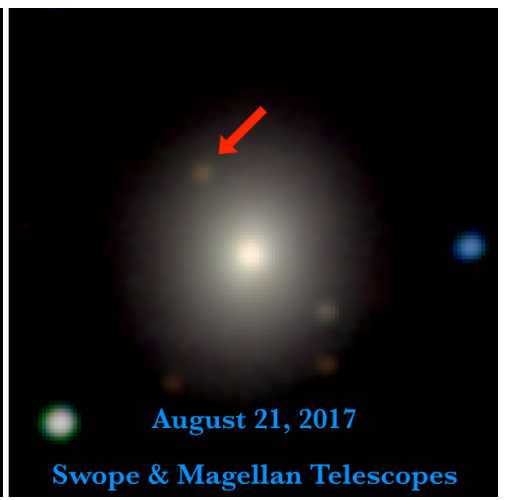

M2H/UC Santa Cruz and Carnegie Observatories/Ryan Foley 
- Duration/relevant time scales

- Methods

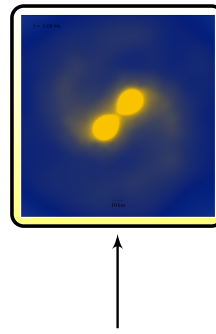

In-spiral:

$\sim \mu \mathrm{s} \rightarrow 30 \mathrm{~s}$

numerical relativity

ET, SpEC, etc.

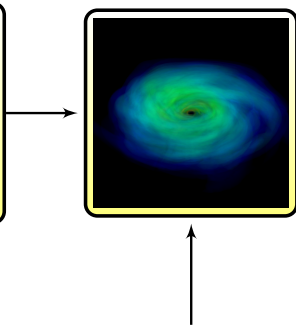

Disk+GRB:

$\sim \mu \mathrm{s} \rightarrow 1 \mathrm{~s}$

GR $\nu \mathrm{RMHD}$

$\nu$ bhlight

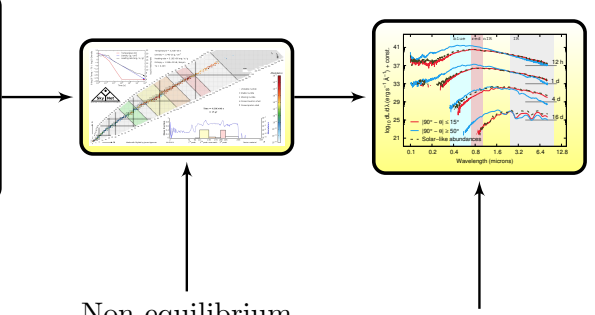

Non-equilibrium reactions:

$\sim \mathrm{s} \rightarrow$ hours $\rightarrow$ Myrs Reaction network PRISM, SkyNet
Photon transport:

$\sim$ Weeks

DDMC-IMC

SuperNu 


\section{Lets Start With the Disk}




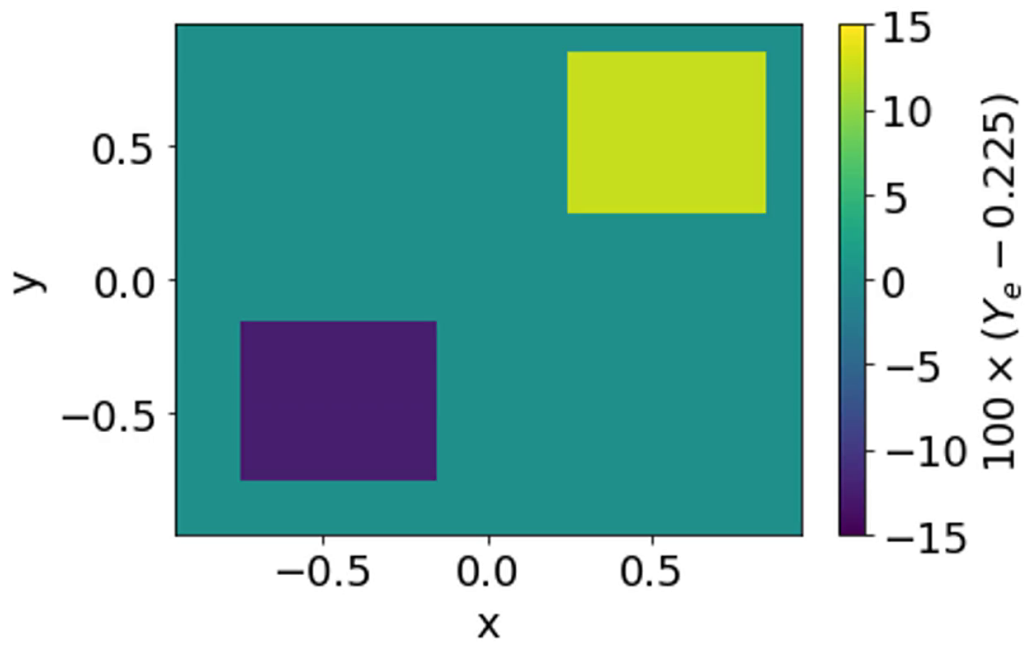

JMM, B. R. Ryan, J. C. Dolence. ApJS 24130 (2019) 


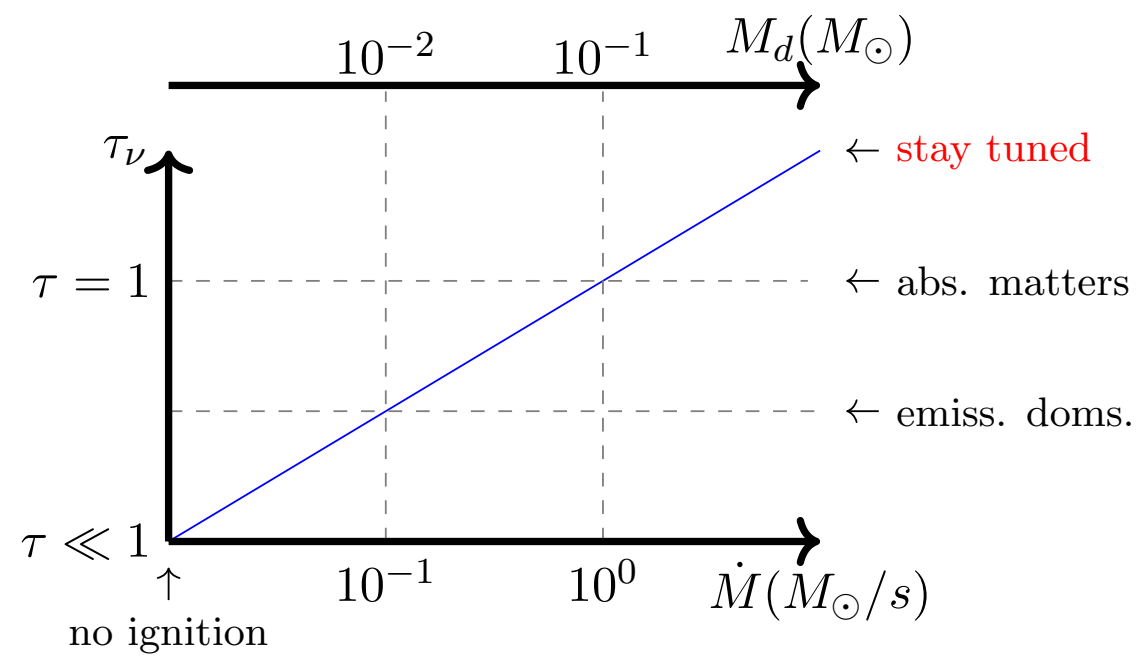




\section{How Much Does Transport Matter?}

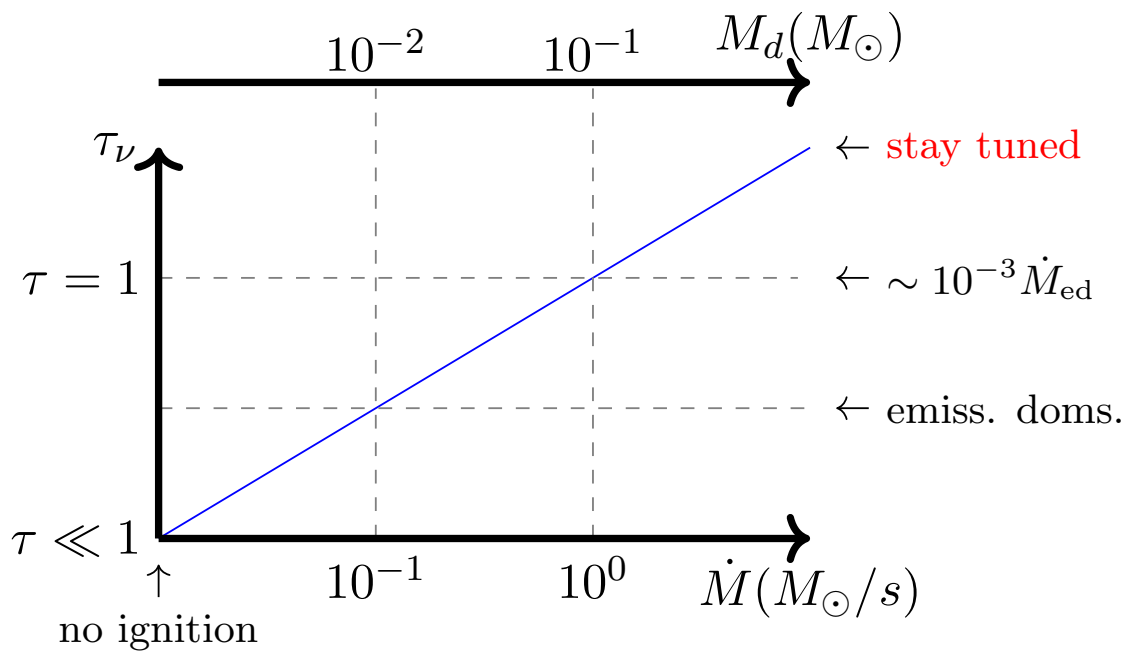




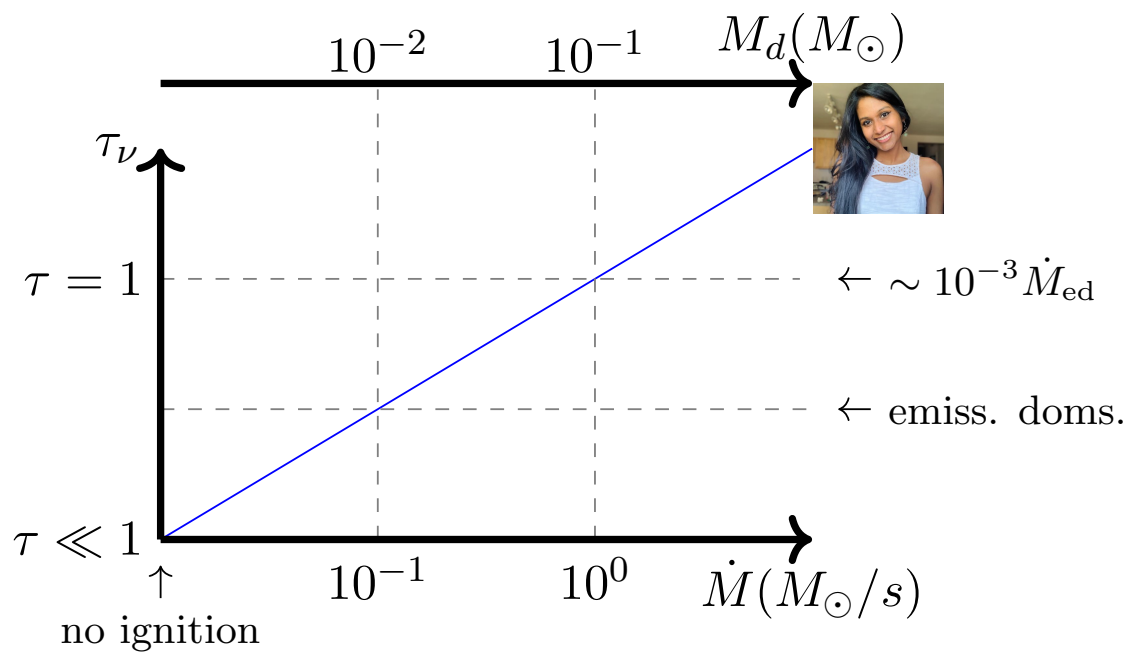


- General relativity

- Rotating black hole spacetime

- Plasma physics

- Ideal magnetohydrodynamics

- Nuclear physics

- Hot gas treated as being in nuclear-statistical equilibrium via equation of state

- Cooling outflow treated in postprocessing via nuclear reaction networks

- Radiation physics

- Material is opaque to photons, can be incorporated in plasma physics

- Material not opaque to neutrinos.

- Neutrinos can change the composition of the material by converting neutrons to protons and vice versa. 
- Mass conservation:

$$
\partial_{t}\left(\sqrt{-g} \rho_{0} u^{t}\right)+\partial_{i}\left(\sqrt{-g} \rho_{0} u^{i}\right)=0
$$

- Momentum and Internal Energy Conservation:

$$
\partial_{t}\left[\sqrt{-g}\left(T^{t}{ }_{\nu}+\rho_{0} u^{t} \delta_{\nu}^{t}\right)\right]+\partial_{i}\left[\sqrt{-g}\left(T^{i}{ }_{\nu}+\rho_{0} u^{i} \delta_{\nu}^{t}\right)\right]=\sqrt{-g}\left(T_{\lambda}^{\kappa} \Gamma_{\nu \kappa}^{\lambda}+G_{\nu}\right)
$$

- Magnetic Fields

$$
\partial_{t}\left(\sqrt{-g} B^{i}\right)-\partial_{j}\left[\sqrt{-g}\left(b^{j} u^{i}-b^{i} u^{j}\right)\right]=0
$$

- Composition

$$
\partial_{t}\left(\sqrt{-g} \rho_{0} Y_{e} u^{t}\right)+\partial_{i}\left(\sqrt{-g} \rho_{0} Y_{e} u^{i}\right)=\sqrt{-g} G_{\mathrm{ye}}
$$

- Neutrino Transport

$$
\frac{D}{d \lambda}\left(\frac{h^{3} I_{\epsilon, f}}{\epsilon^{3}}\right)=\left(\frac{h^{2} \eta_{\epsilon, f}}{\epsilon^{2}}\right)-\left(\frac{\epsilon \chi_{\epsilon, f}}{h}\right)\left(\frac{h^{3} I_{\epsilon, f}}{\epsilon^{3}}\right),
$$


- General relativistic radiation magnetohydrodynamics for kilonova disks

- Open Source! https://github.com/LANL/nubhlight

- Magnetized gas via finite volume methods

- Standard second-order Gudonov scheme

- Cell-centered constrained transport for magnetic fields

- WENO5 reconstruction

- Local Lax-Friedrichs Riemann solver

- Neutrinos via Monte Carlo methods

- Explicit integration along geodesics

- Probabilistic emissivity, absorption, and scattering

- Novel biasing scheme ensures all processes well-sampled

- Coupled via operator splitting

- Built on top of HARM, grmonty, and bhlight. 


\section{The August 2017 Disk}
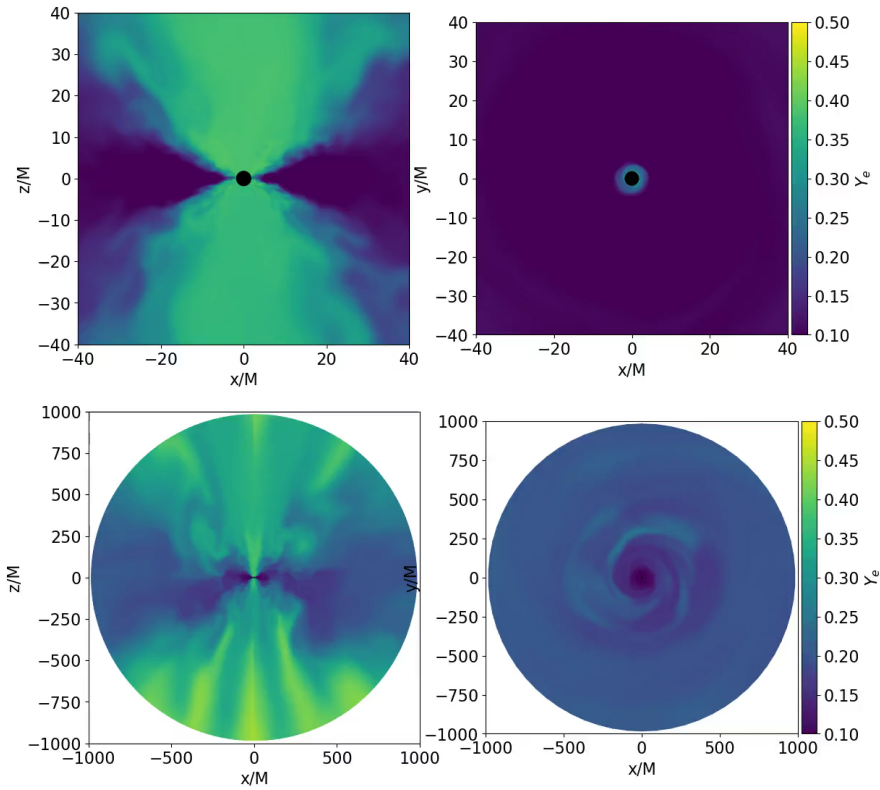


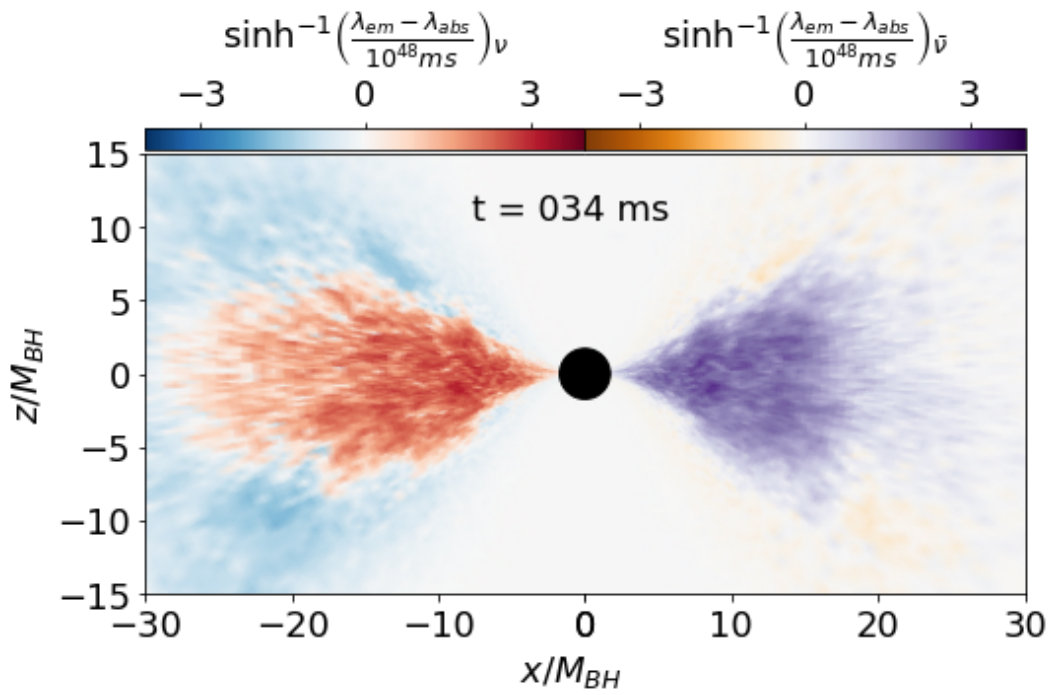

JMM et al. PRD 100023008 (2019) 


\section{Electron Fraction of the Outflow}

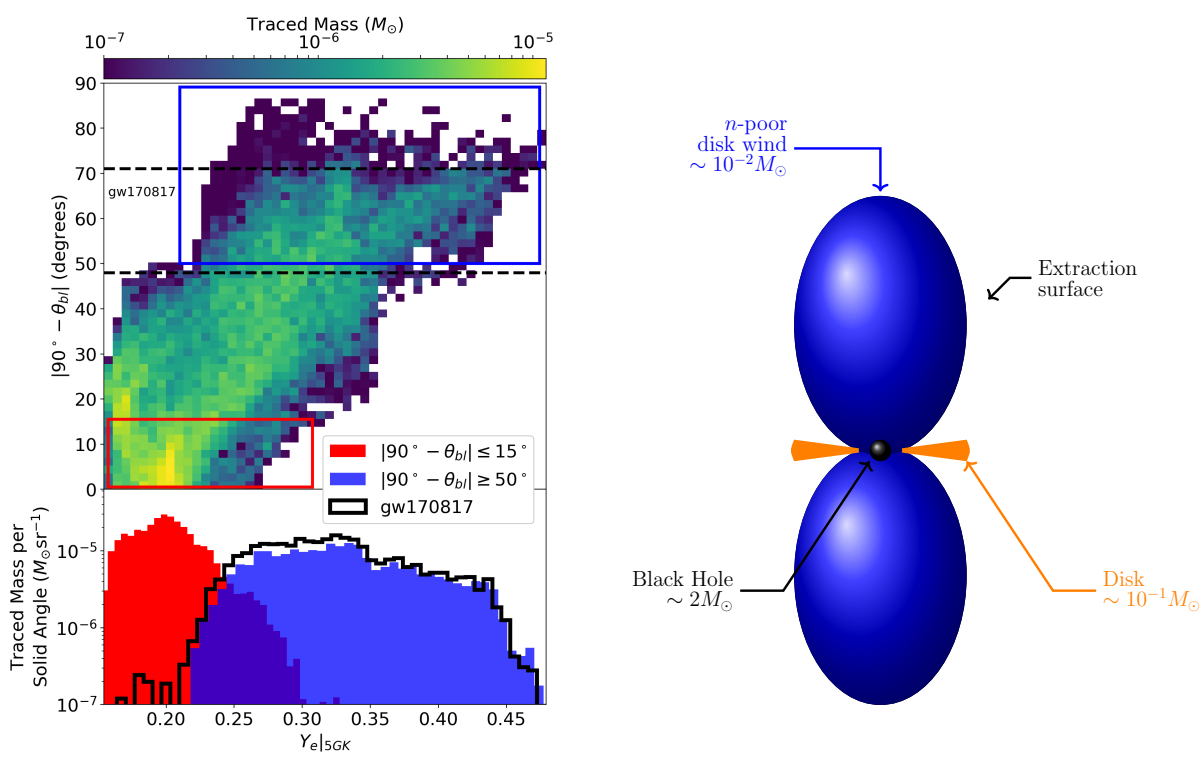

JMM et al. PRD 100023008 (2019) 


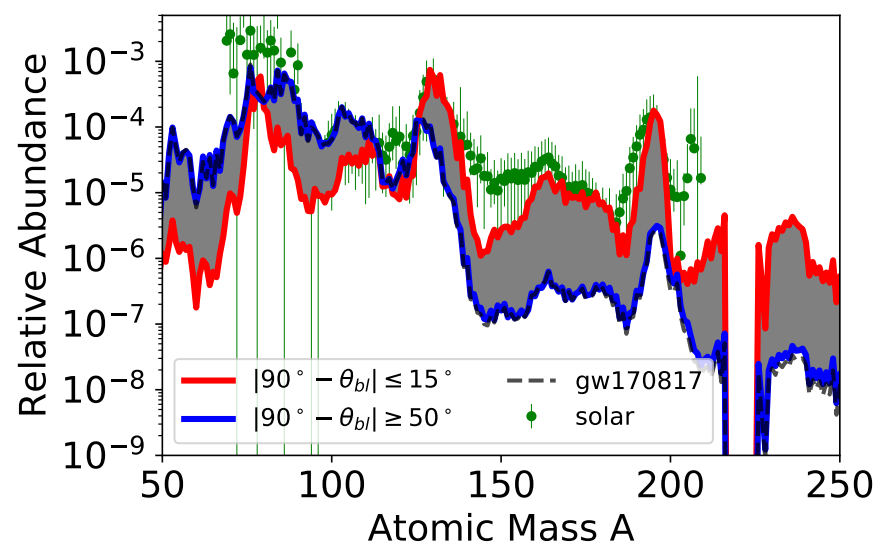

- r-process networks:

- SkyNet

- PRISM

- CFNET

- etc.

JMM et al. PRD 100023008 (2019) 


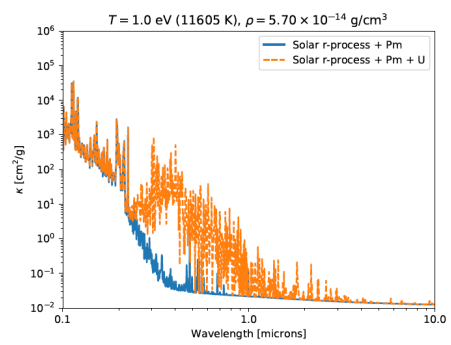

Even,..,JMM, et al. ApJ 89924 (2020)

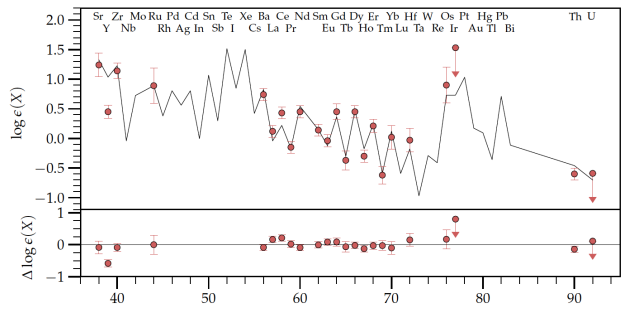

Cain et al. ApJ 89840 (2020) 


\section{A Sampling of Recent Progress (Not my work)}

Fission Yields
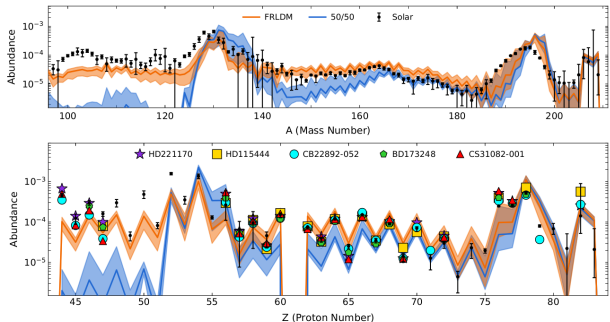

Vassh et al., ApJ 89628 (2020)

Heating rates + mass models

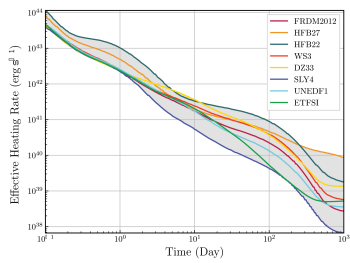

Zhu et al., ApJ 906 94, (2021)

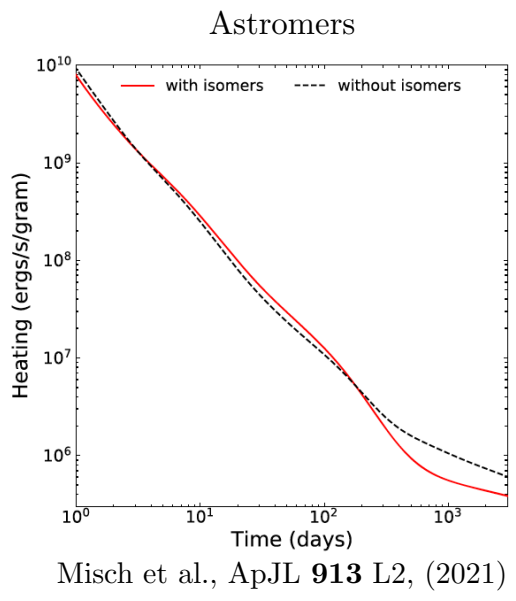




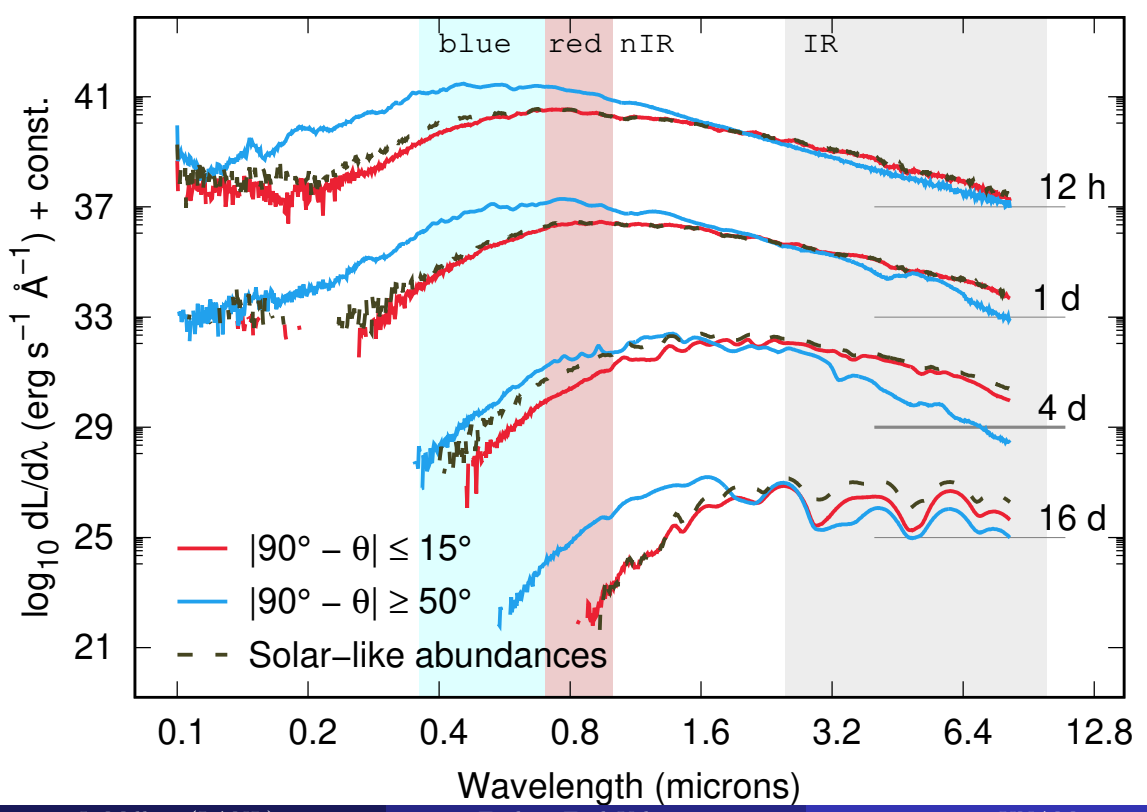




\section{How Opacities are Computed (Not my work)}

- Atomic lines determine spectrum

- Lines determined by energy levels, wavefunction, matrix elements

- Usually modeled assuming LTE

- LANL atomic modeling codes: Fontes et al., JPB 48 144014, (2015)

- LANL LTE opacities: Fontes et al., MNRAS 4934143 (2020)

- Lanthanide opacities available at https://nlte.nist.gov/OPAC

- Experimental atomic data badly needed

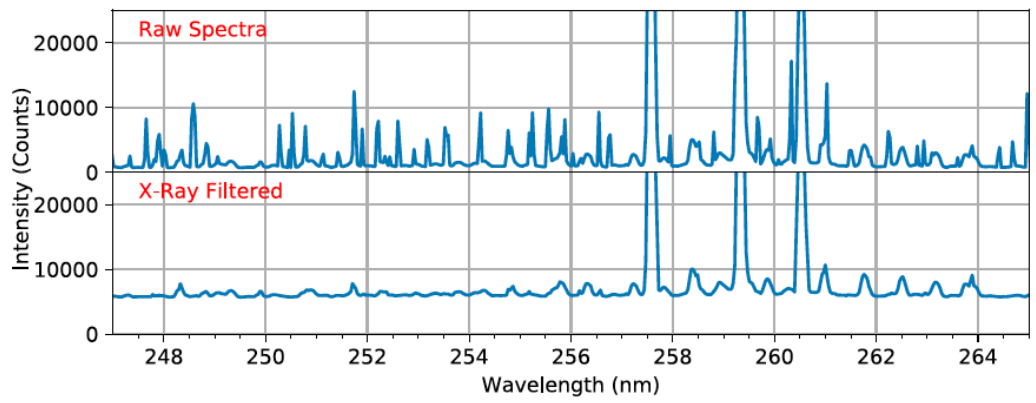

Bromley et al., ApJS 250 19, (2020). 


\section{Outflows, Nucleosynthesis, Observables}

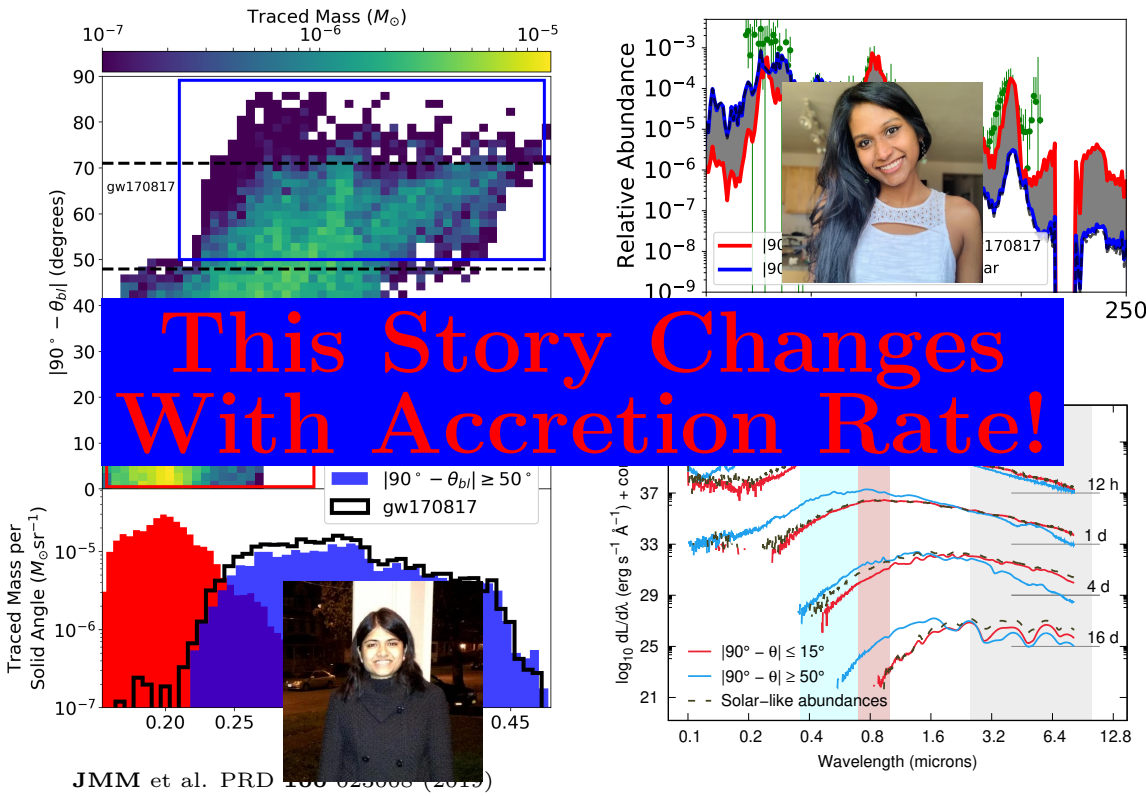


- Accretion times $t \sim 10 \mathrm{~s}$

- $\dot{M}$ between

$$
\begin{aligned}
& \text { - } 10^{-4} M_{\odot} / s \\
& \text { - } 10^{-1} M_{\odot} / s
\end{aligned}
$$

- $\rho \sim 10^{10} \mathrm{~g} / \mathrm{cm}^{3}$

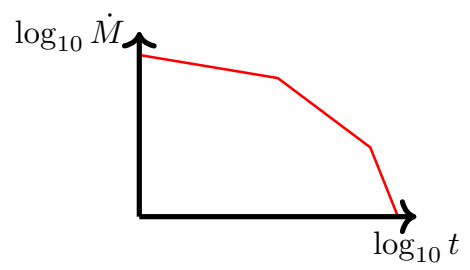

Siegel, Barnes, Metzger. Nature 241 (2019)

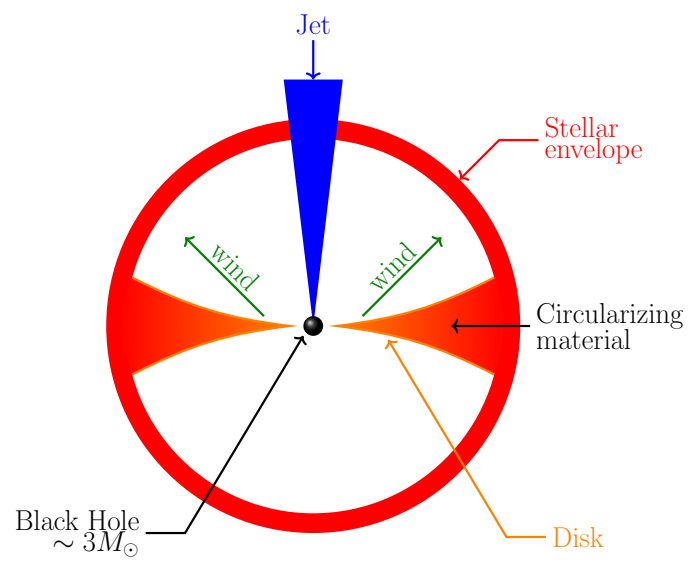




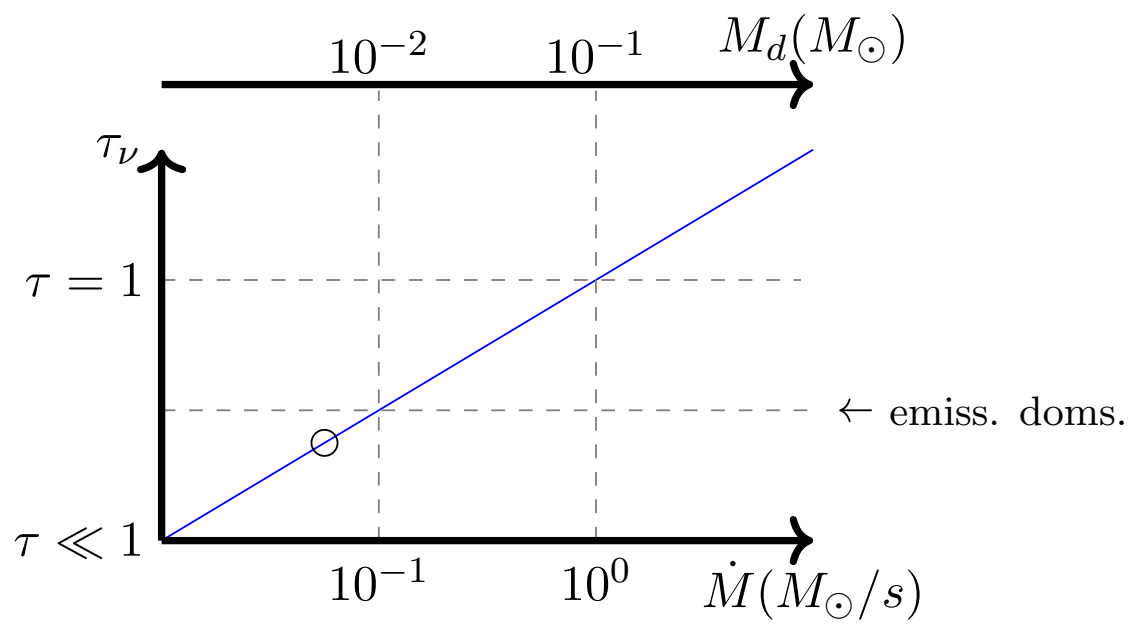




\section{Building a Collapsar Disk}
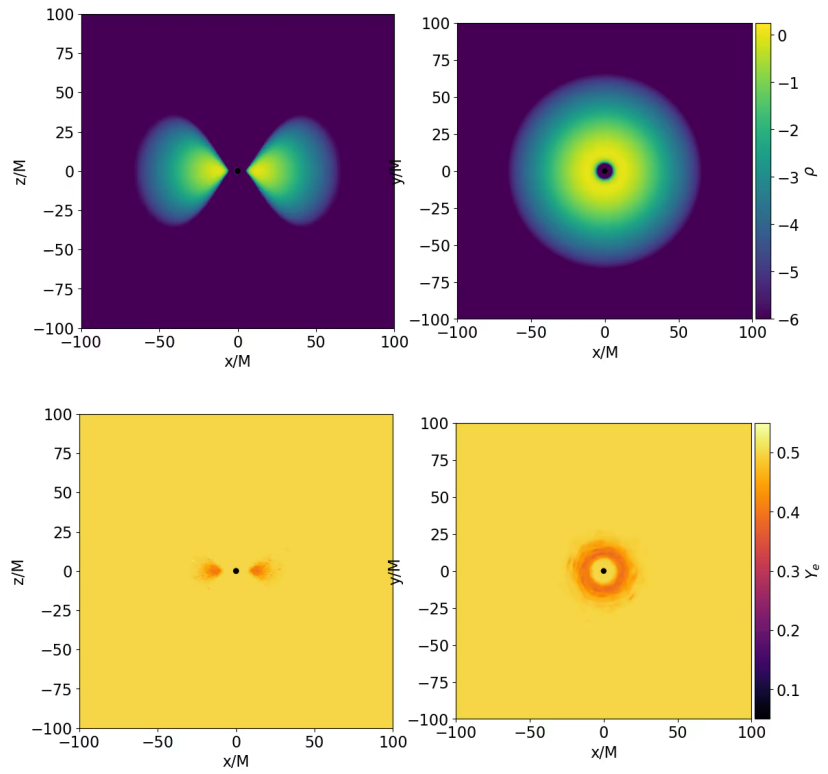


\section{Building a Collapsar Disk}
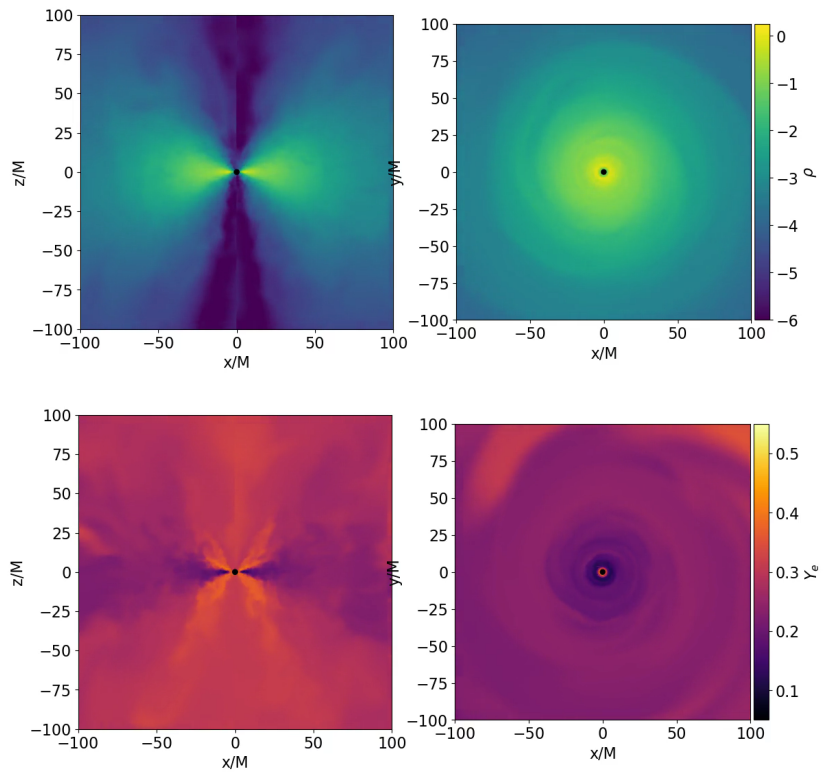


\section{Stationary Disk, No Ye equilibrium!}
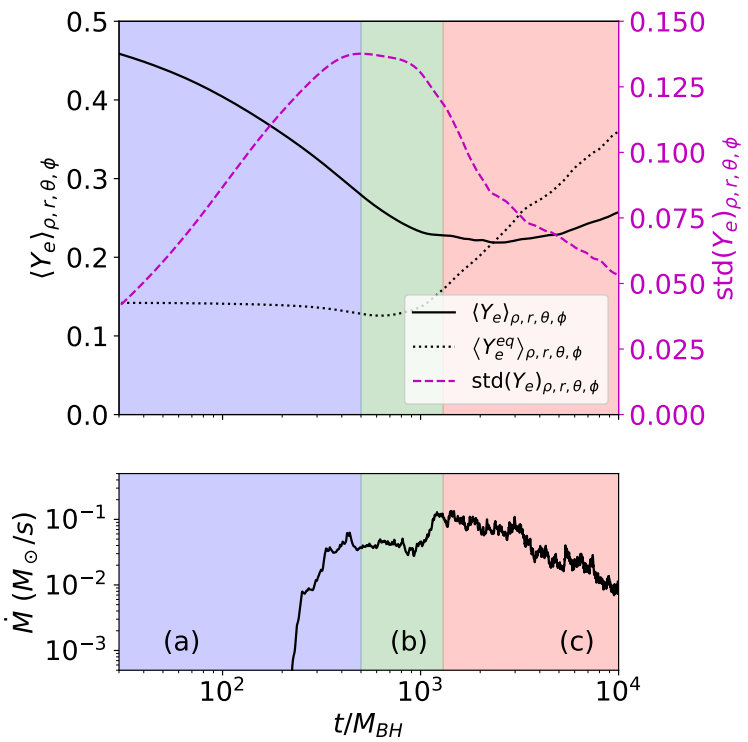


\section{Stationary Disk, No Ye equilibrium!}

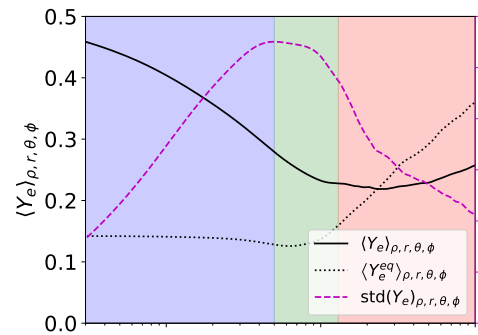

0.150
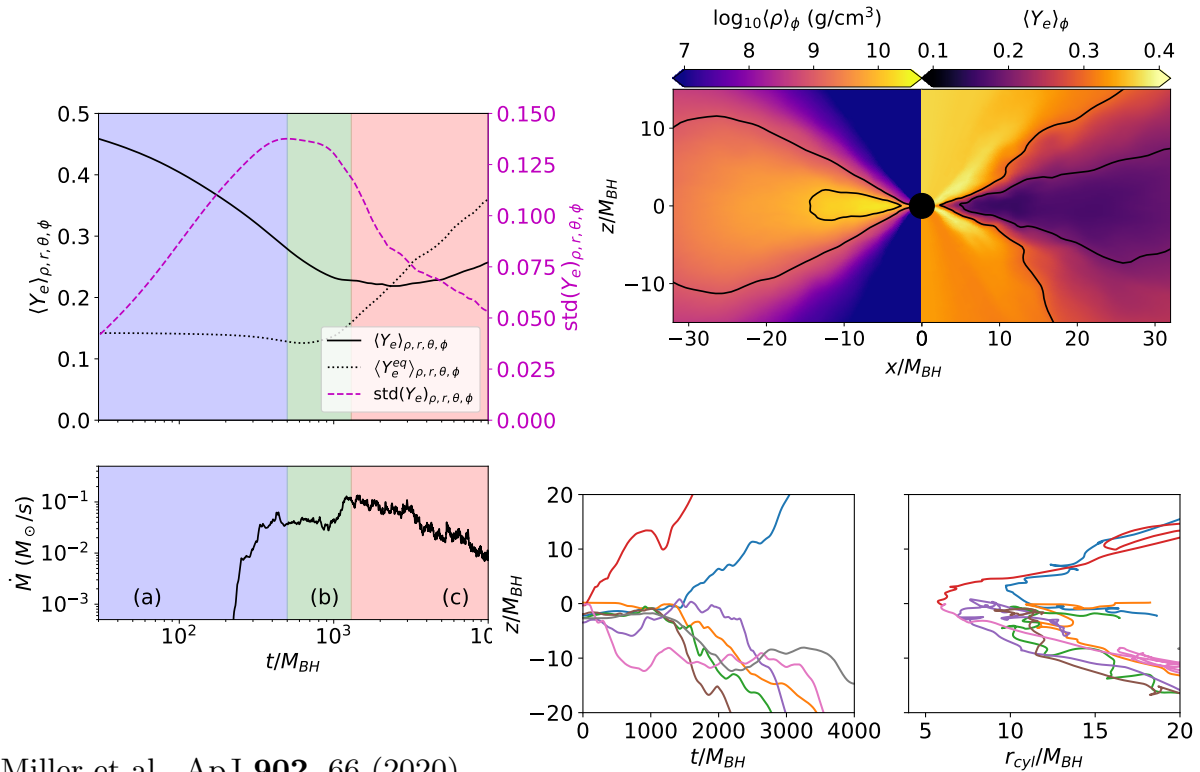

Miller et al., ApJ 902, 66 (2020) 


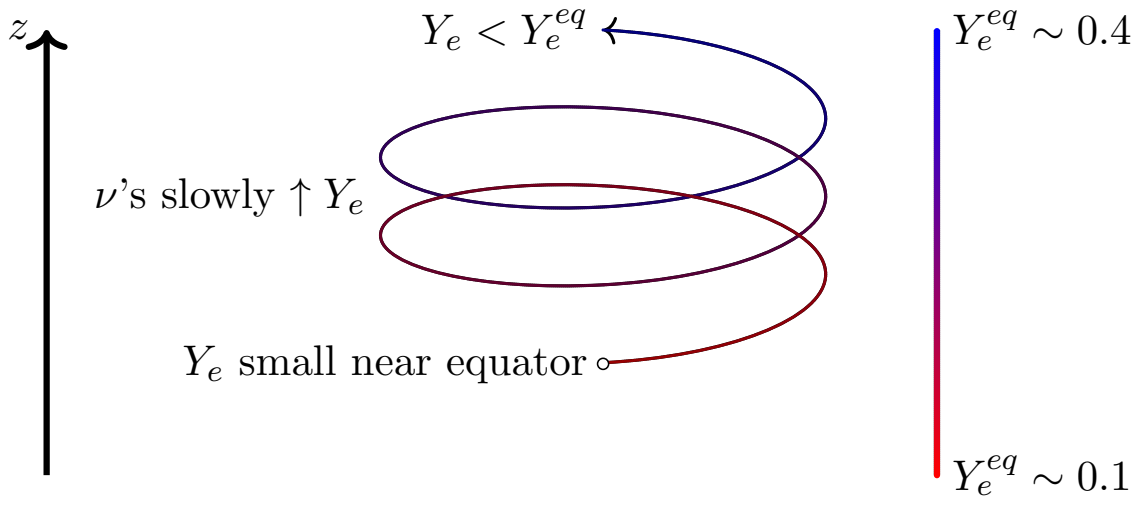


$Y_{e}$ is set by the balance of Turbulence and Neutrinos!

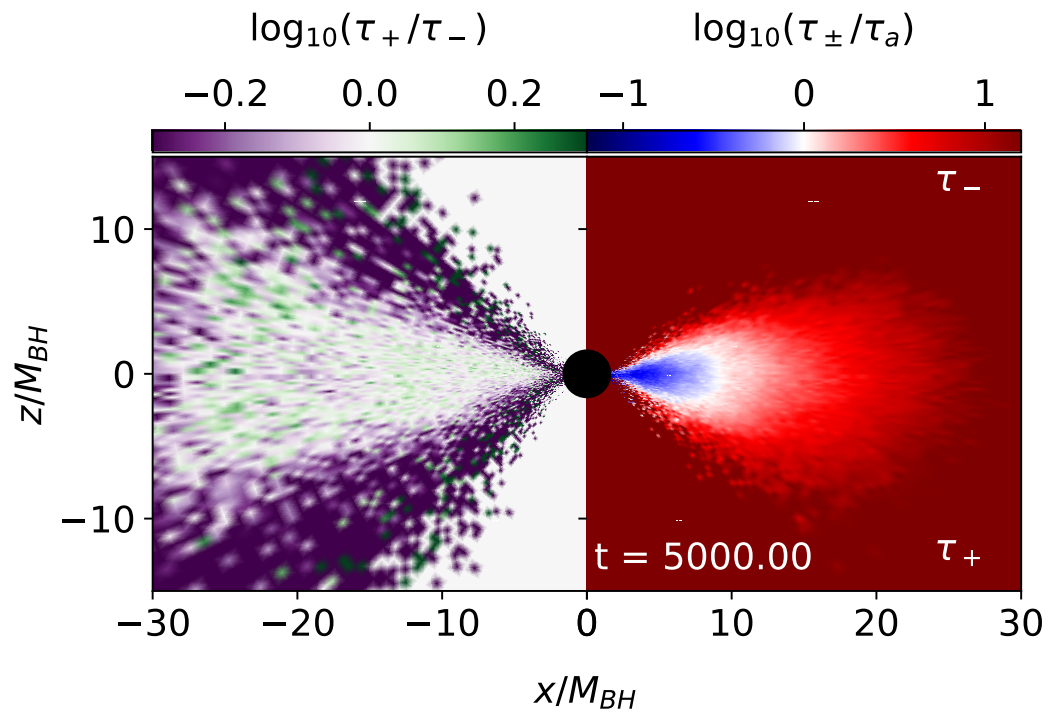

Miller et al., ApJ 902, 66 (2020) 


$$
Y_{\mathrm{e}}(z / H)=\left\langle\min \left(Y_{\mathrm{e}}\right)\right\rangle_{\mathrm{trc}}+\left\langle\frac{d Y_{\mathrm{e}}}{d t}\right\rangle_{t, \operatorname{trc}}\left(H\left\langle\frac{d z}{d t}\right\rangle_{t, \operatorname{trc}}^{-1}\right)\left(\frac{z}{H}-\langle\min (z / H)\rangle_{\operatorname{trc}}\right)
$$
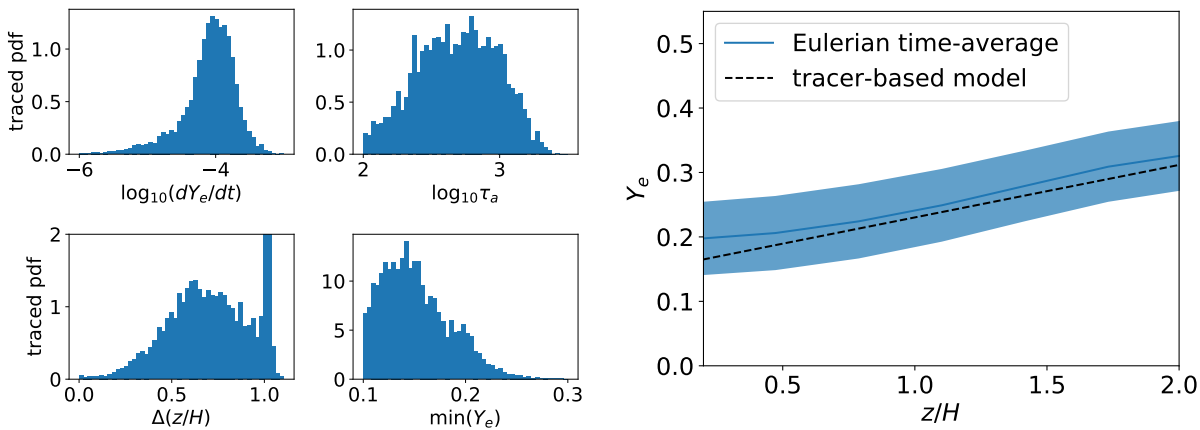

Miller et al., ApJ 902, 66 (2020) 


\section{Take-home Messages}
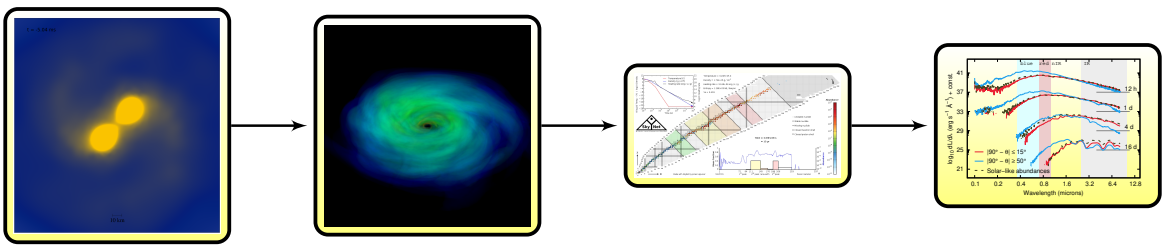

- Neutron star mergers are awesome!

- Source of GRBs, heavy elements, kilonova afterglow, gravitational waves

- Despite huge successes so far, connecting an observation to an astrophysical system is complicated and challenging:

- Involves all four fundamental forces, many different physical processes, modeled by very different codes/capabilities

- Many degeneracies between astrophysical uncertainty, microphysical uncertainty, etc.

- Neutrino transport essential to get right in the disk. Many open questions remain. 


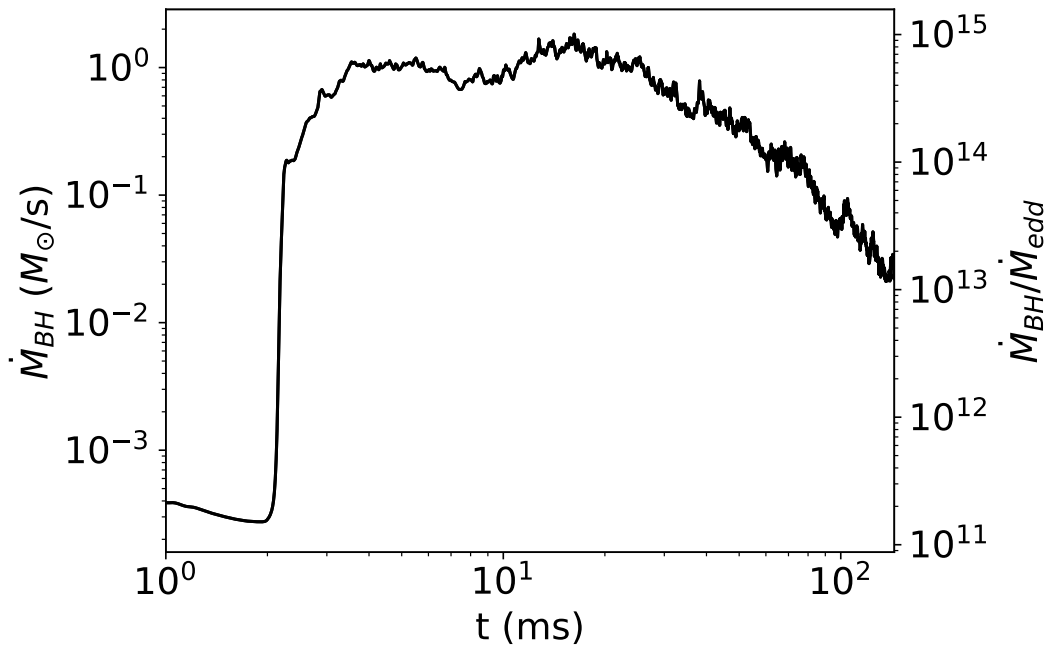




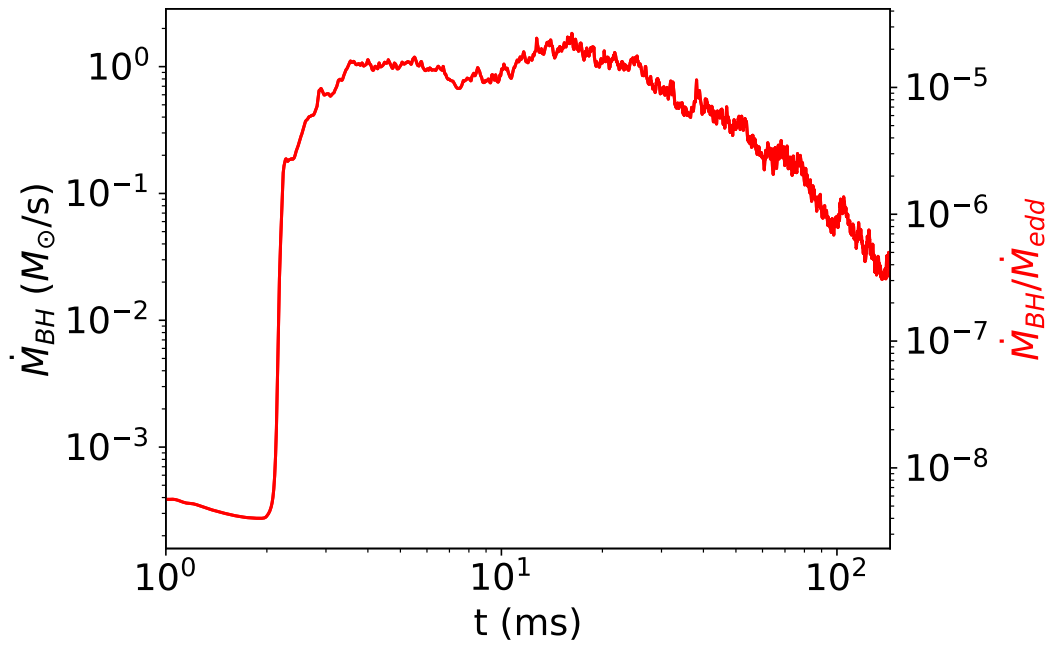




\section{Neutrino Transport}
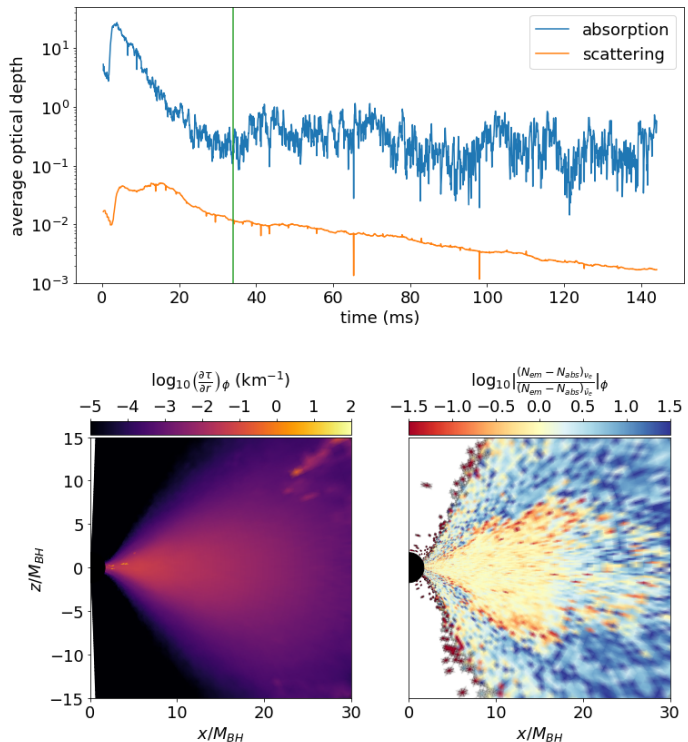


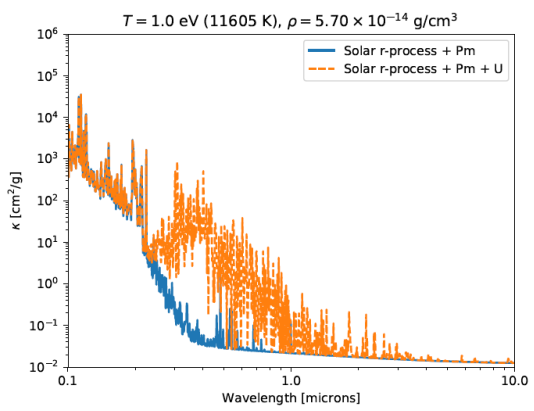

- Not all opacities known, so surrogates often used. Some elements matter more than others.

arXiv:1904.13298

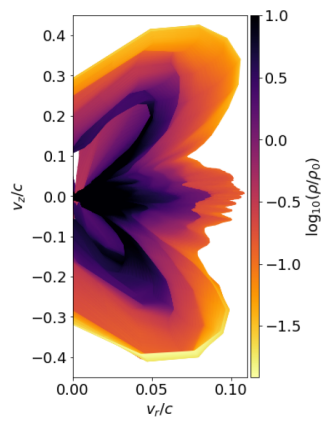

- Geometric effects can be significant, are difficult to treat, and are degenerate with other parameters, such as ejecta mass.

arXiv:204.00102 
- Large optical depths, such as inside a neutron star present issues for Monte Carlo

- Need a method that can span the range of optical depths and solve the full transport equation

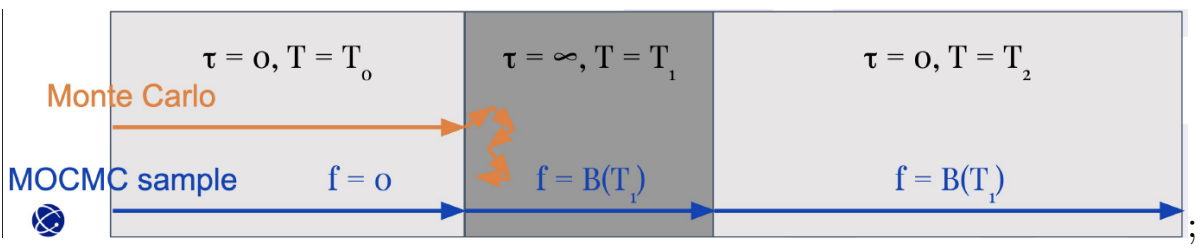

